\title{
ACCION ANTIFUNGICA DE LAS ASOCIACIONES DE SULFAMETOXAZOL O KETOCONAZOL CON ETOSUCCIMIDA, FENILBUTAZONA, IBUPROFENO, GENTAMICINA, METAMIZOL, YODURO DE POTASIO Y OTROS FARMACOS.
}

Waldo Lazo

Departamento de Ciencias Ecológicas

Facultad de Ciencias, Universidad de Chile.

Palabras clave: Antifúngico, sinergismo, sulfametoxazol, etosuccimida, fenilbutazona, gentamicina, ibuprofeno ketoconazol, metamizol, yoduro de potasio.

Key words: Antifungal, sinergism, sulphametoxazole, ethosuximide, phenylbutazone, ibuprofen, gentamycin, ketoconazole, metamizol, potassium iodide,

\section{RESUMEN:}

Se obtuvo la inhibición total del desarrollo fúngico in vitro durante 48 o más horas, al agregar al medio de cultivo los fármacos siguientes: para Acremonium potronii $y$ A. falciforme $50 \mathrm{ug} / \mathrm{ml}$ de sulfametoxazol; para Fusarium solanii sulfametoxazol $100 \mathrm{ug} / \mathrm{ml}$ más fenilbutazona $30 \mathrm{ug} / \mathrm{ml}$ más etosuccimida $100 \mathrm{ug} / \mathrm{ml}$ o sulfametoxazol $100 \mathrm{ug} / \mathrm{ml}$ más ibuprofeno $30 \mathrm{ug} / \mathrm{ml}$ más etosuccimida $100 \mathrm{ug} / \mathrm{ml}$; para Candida albicans y Aspergillus niger ketoconazol $0.5 \mathrm{ug} / \mathrm{ml}$ más gentamicina $3-10 \mathrm{ug} / \mathrm{ml} ;$ para A. fumigatus sulfametoxazol $100 \mathrm{ug} / \mathrm{ml}$ más yoduro de potasio $100 \mathrm{ug} / \mathrm{ml}$ más metamizol $100 \mathrm{ug} / \mathrm{ml}$ o sulfametoxazol $80-100 \mathrm{ug} / \mathrm{ml}$ más fenilbutazona 20 $30 \mathrm{ug} / \mathrm{ml}$ más etosuccimida $100 \mathrm{ug} / \mathrm{ml} ;$ o sulfametoxazol $80 \mathrm{ug} / \mathrm{ml}$ más clorpromacina $10 \mathrm{ug} / \mathrm{ml}$.

\section{INTRODUCCION}

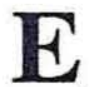

n los últimos doce años han aparecido numerosos fármacos de acción antifúngica, muchos de los cuales se emplean con éxito en clínica (Martindale 1982, Negroni 1983, Goodman Gilman 1985, Lazo y Ferrada 1986, Saag y Dismukes 1988) para el tratamiento tópico o sistémico de diversas micosis. Se ha encontrado tam-

\section{SUMMARY}

[Antifungal action of the associations among sulphametoxazole or ketoconazole and ethosuccimide, phenylbutazone, ibuprofen, gentamycin, metamizol, potassium iodide and other drugs.]

The total inhibition of in vitro fungal growth during 48 or more hours was obtained by the addition to the culture media of the following drugs: for Acremonium potronii and Acremonium falciforme 50 $\mathrm{ug} / \mathrm{ml}$ of sulphametoxazole; for Fusarium solanii sulphametoxazole $100 \mathrm{ug} / \mathrm{ml}$ plus phenylbutazone 30 $\mathrm{ug} / \mathrm{ml}$ plus ethosuccimide $100 \mathrm{ug} / \mathrm{ml}$ or sulphametoxazole $100 \mathrm{ug} / \mathrm{ml}$ plus ibuprofen $30 \mathrm{ug} / \mathrm{ml}$ plus ethosuccimide $100 \mathrm{ug} / \mathrm{ml}$; for Candida albicans and Aspergillus niger ketoconazole $0.5 \mathrm{ug} / \mathrm{ml}$ plus gentamycin 3-10 ug/ml; for A. fumigatus sulphametoxazole $100 \mathrm{ug} / \mathrm{ml}$ plus potassium iodide $100 \mathrm{ug} / \mathrm{ml}$ plus metamizol $100 \mathrm{ug} / \mathrm{ml}$ or sulphametoxazole 80$100 \mathrm{ug} / \mathrm{ml}$ plus phenylbutazone $20-30 \mathrm{ug} / \mathrm{ml}$ plus ethosiuccimide $100 \mathrm{ug} / \mathrm{ml}$ or sulphametoxazole 80 $\mathrm{ug} / \mathrm{ml}$ plus chlorpromazine $10 \mathrm{ug} / \mathrm{ml}$

bién que dos fenotiacinas: la trifluoperacina y la clorpromacina inhiben el desarrollo in vitro de algunas levaduras (Eilam y col. 1987) y que ciertos antifúngicos si se asocian con antibacterianos incrementan su acción antimicótica (Lazo y Ferrada 1986, Antunez y col. 1986).

Diversos autores han señalado la acción antifúngica de las sulfamidas (Copeland y Rosvoll 1969, Kamaya 1973, Lazo y Ferrada 1986) y desde 1940 se ha usado sulfamidas en el tratamiento clínico de algunas micosis (Negroni 1983). 
En el presente ensayo se estudió la acción antifúngica in vitro de algunos fármacos antimicrobianos frente a diversos hongos y luego se investigó la acción de las asociaciones binarias o ternarias de esos fármacos con muchos otros. A algunos de esos fármacos se los investigó, pues, por razones de orden clínico se los suele prescribir al mismo tiempo que los antimicóticos, como analgésicos o antiinflamatorios y se deseaba conocer si su asociación afectaba de algún modo la acción in vitro del antifúngico.

\section{MATERIALES Y METODOS}

Se estudió la inhibición del desarrollo in vitro de cepas de Candida albicans, Aspergillus fumigatus, A. niger, Acremonium falciforme, A. potronii, Fusarium solani. Para cultivar estos hongos se empleó un medio que contenía polvo nutriente Gibco 4 gramos, dextrosa 10 gramos, agua destilada $1000 \mathrm{ml}$. Cuando se quizo utilizar un medio sólido se agregó al anterior 14 gramos de agar en polvo por litro.

También se usó un medio a base de polvo nutriente Gibco 8 gramos, dextrosa 10 gramos, agua destilada $1000 \mathrm{cc}$.

A frascos que contenían $50 \mathrm{ml}$ del medio nutriente se les añadio los fármacos o asociaciones farmacológicas deseadas y el inóculo del hongo respectivo, que consistió en $1 \mathrm{ml}$ de una solución que contenía 200.000 unidades formadoras de colonias, luego se añadió $50 \mathrm{ml}$ del medio agar nutriente fundido, se agitó la mezcla y se vació 15 o 20 $\mathrm{ml}$ de ella en cada placa de Petri. Una vez que el agar se hubo solidificado se colocó las placas en una estufa a $35^{\circ} \mathrm{C}$ durante 48 a 96 horas.

También se realizaron estudios con inóculos 30 más veces superiores al anteriormente indicado.

Como fuente de inóculo se usó cultivos de hongos miceliados de 2 a 4 semanas de antigüedad y cultivos de $\mathbb{C}$. albicans de 20 a 24 hrs.

Las asociaciones farmacológicas fueran binarias o ternarias tuvieron como fármacos base, el sulfametoxazol o el ketoconazol y fue la acción antifúngica de cada uno de estos fármacos la que se trató de potenciar mediante la asociación con los otros fármacos. Para cumplir este propósito fue preciso usar concentraciones subinhibitorias de ketoconazol y sulfametoxazol y comprobar si al añadir los otros fármacos la asociación había logrado inhibir, parcial o totalmente, el desarrollo fúngico.

Los fármacos cuya posible acción antimicótica directa o en asociación con otros- se estudió fueron: ambroxol, acetaminofeno, ácido acetil salicílico, ácido para-amino-benzoico, ácido para-amino- salicilico, ácido nalidíxico, amicacina, aminofilina, amitriptilina, amoxicilina, ampicilina, aztreonam, bencilpenicilina, carbamacepina, cefazolina, cefradina, ceftizoxima, ceftriaxona, clindamicina, cloranfenicol, cloroquina, clorpromacina, colistin, diyodo-hidroxiquinolina, doxiciclina, etionamida, eritromicina, etosuccimida, fosfomicina, fenitoína, fenilbutazona, gentamicina, griseofulvina, ibuprofeno, imipramina, isoniacida, kanamicina, ketoconazol, metamizol, metronidazol, neomicina, nitrofurantoína, nifurtimox, norfloxacina, piperacina, piracinamida, quinina, rifampicina, sulfatiazol sulfametoxazol, sulfametoxipiridacina, tetraciclina, tinidazol, tiacetazona, tianfenicol, trimetoprim, yodoclorhidroxiquinolina, yoduro de potasio.

Para disolver los fármacos no hidrosolubles se usó etanol absoluto o dimetilsulfóxido. A los controles se les añadio también la cantidad respectiva del solvente empleado. Todos los experimentos se hicieron en duplicado

\section{RESULTADOS}

Las asociaciones binarias o ternarias de los 58 fármacos que se probaron, ascendieron a varios cientos y se observó que la inmensa mayoría de ellas no ejercía acción inhibitoria alguna sobre el desarrollo de las especies fúngicas usadas; unas pocas asociaciones inhibieron parcial y temporalmente ese desarrollo, pero, sólo las que a continuación se detallan lo inhibieron totalmente durante 48 a 96 horas cuando se utilizó el medio de cultivo que contenía $4 \mathrm{~g} / 1$ de polvo nutriente Gibco y 200.000 unidades formadoras de colonia como inóculo. En el medio con $8 \mathrm{~g} / 1$ de polvo nutriente o con inóculos de 600.000 o más UFC no hubo inhibición del desarrollo fúngico.

De las tres sulfamidas ensayadas la sulfametoxipiridacina no ejerció acción antifúngica directa. $\mathrm{La}$ adición de 2 a $10 \mathrm{ug} / \mathrm{ml}$ de trimetoprim no potenció ni antagonizó la acción antifúngica del sulfametoxazol.

El sulfametoxazol en concentraciones de 50 $\mathrm{ug} / \mathrm{ml}$ inhibió completamente durante 72 a 96 horas el desarrollo de A. falciforme y A. potronii, pero $A$. fumigatus y $\mathbb{F}$. solani fueron inhibidos sólo en forma parcial durante 48 horas, aún en concentraciones de $140 \mathrm{ug} / \mathrm{ml}$. La asociación binaria de sulfametoxazol con etosuccimida, metamizol, yoduro de potasio o fenilbutazona, aunque aumenta su acción fungistática no basta para hacerla total. Sólo se consiguió la inhibición completa del desarrollo fúngico durante 48 a 96 horas, al emplear las asociaciones farmacológicas siguientes: para A.fumigatus sulfametoxazol $80 \mathrm{ug} / \mathrm{ml}$ más fe- 
nilbutazona $30 \mathrm{ug} / \mathrm{ml}$ más etosuccimida $100 \mathrm{ug} / \mathrm{ml}$ o sulfametoxazol $100 \mathrm{ug} / \mathrm{ml}$ más fenilbutazona 20 $\mathrm{ug} / \mathrm{ml} \mathrm{más} \mathrm{etosuccimida} 100 \mathrm{ug} / \mathrm{ml}$ (ver Tabla 1) (foto $1 . \mathrm{N}^{2} 1$ y 2 ), o sulfametoxazol $100 \mathrm{ug} / \mathrm{ml}$ más yoduro de potasio $100 \mathrm{ug} / \mathrm{ml}$ más metamizol 100 ug/mI o sulfametoxazol $80 \mathrm{ug} / \mathrm{ml}$ más clorpromacina $10 \mathrm{ug} / \mathrm{ml}$. Para F.solani sulfametoxazol 100 $\mathrm{ug} / \mathrm{ml}$ más fenilbutazona $30 \mathrm{ug} / \mathrm{ml}$ más etosuccimida $100 \mathrm{ug} / \mathrm{ml}$ (foto 1 . № 3 y 4 ).

El ketoconazol inhibió el desarrollo de las cepas de C. albicans, A. fumigatus, A. niger y F. solani estudiadas, sólo en concentraciones de 3 a 12 $\mathrm{ug} / \mathrm{ml}$, por lo tanto para investigar la posible acción potenciadora de los otros fármacos, se utilizó el ketoconazol en concentraciones sub-inhibitorias de $0.5 \mathrm{a} 1 \mathrm{ug} / \mathrm{ml}$. Al proceder en esta forma se encontró que la asociación de $0.5 \mathrm{ug} / \mathrm{ml}$ de ketoconazol más 3-10 ug/ml de gentamicina inhibía totalmente el desarrollo de varias cepas de C. albicans y A. niger cuya CIM para el ketoconazol fluctuaba entre 4-10 ug/ml y para la gentamicina entre 50 a $120 \mathrm{ug} / \mathrm{ml}$. Ninguna otra asociación binaria o ternaria de ketoconazol -en concentraciones sub-inhibitorias- con alguno de los 58 fármacos antes nombrados consiguió inhibir totalmente el desarrollo de C. albicans, A. fumigatus, A. niger y $\mathrm{F}$. solani.

El resumen de los resultados obtenidos se expresa en la Tabla 1.

Tabla № 1

Fármacos o asociaciones farmacológicas

Hongos cuyo desarrollo se empleadas inhibió totalmente durante 48 a 96 horas ...

\begin{tabular}{ll}
\hline sulfametoxazol $50 \mathrm{ug} / \mathrm{ml}$ & A. falciforme, A. potronii \\
\hline $\begin{array}{l}\text { sulfametoxazol } 100 \mathrm{ug} / \mathrm{ml}+\text { fenilbutazona } \\
30 \mathrm{ug} / \mathrm{ml}+\text { etosuccimida } 100 \mathrm{ug} / \mathrm{ml}\end{array}$ & F. solani \\
\hline $\begin{array}{l}\text { sulfametoxazol } 100 \mathrm{ug} / \mathrm{ml}+\mathrm{ibuprofeno} \\
30 \mathrm{ug} / \mathrm{ml}+\text { etosuccimida } 100 \mathrm{ug} / \mathrm{ml}\end{array}$ & F. solani \\
\hline $\begin{array}{l}\text { sulfametoxazol } 80 \mathrm{ug} / \mathrm{ml}+\text { fenilbutazona } \\
30 \mathrm{ug} / \mathrm{ml}+\text { etosuccimida } 100 \mathrm{ug} / \mathrm{ml}\end{array}$ & A. fumigatus \\
\hline $\begin{array}{l}\text { sulfametoxazol } 100 \mathrm{ug} / \mathrm{ml}+\text { fenilbutazona } \\
20 \mathrm{ug} / \mathrm{ml}+\text { etosuccimida } 100 \mathrm{ug} / \mathrm{ml}\end{array}$ & A. fumigatus \\
\hline $\begin{array}{l}\text { sulfametoxazol } 80 \mathrm{ug} / \mathrm{ml}+\text { clorpromacina } \\
10 \mathrm{ug} / \mathrm{ml}\end{array}$ & A. fumigatus \\
\hline $\begin{array}{l}\text { sulfametoxazol } 100 \mathrm{ug} / \mathrm{ml}+\text { yoduro de } \\
\text { potasio } 100 \mathrm{ug} / \mathrm{ml}+\mathrm{metamizol} 100 \mathrm{ug} / \mathrm{ml}\end{array}$ & A. fumigatus \\
\hline $\begin{array}{l}\text { ketoconazol } 0.5 \mathrm{ug} / \mathrm{ml}+\text { gentamicina } \\
\text { 3-10 ug/ml }\end{array}$ & $\begin{array}{l}\text { C. albicans, } \\
\text { A. niger }\end{array}$ \\
\hline
\end{tabular}

\section{COMENTARIO}

Ninguna de las asociaciones ternarias de sulfametoxazol con fenilbutazona y etosuccimida o yoduro de potasio y metamizol fue fungicida y sólo se consiguió inhibiciones totales temporales del desarrollo fúngico.
La concentración de los inóculos demostró también ser decisiva en los resultados de estas pruebas de inhibición. Con inóculos cuya concentración era 3 o más veces superior a la que se usó (200.000 UFC/ml) los períodos de inhibición total se acortaban a 36 horas o no había inhibición total.

También se comprobó que la composición del medio de cultivo podía influir sobre los resultados. 
Si se aumentaba a 8 gramos por litro la cantidad de polvo nutriente, no había inhibición o se necesitaba dosis superiores de sulfametoxazol y de los otros fármacos para conseguir la inhibición del desarrollo fúngico.

Como los antifúngicos de uso sistémico: anfotericina $\mathrm{B}$, miconazol y ketoconazol, no atraviesan la barrera hematoencefálica, ni siquiera con las meninges inflamadas, ni tampoco alcanzan concentraciones significativas en los humores acuoso o vítreo oculares (lo cual dificulta decisivamente el tratamiento de las endoftalmitis fúngicas o de las micosis del sistema nervioso central), la acción antifúngica del sulfametoxazol, potenciada por otros fár- macos podría ser de interés, ya que el sulfametoxazol atraviesa la barrera hemato-enfálica alcanzando concentraciones útiles en el líquido céfalo raquídeo, así como también las alcanza en los humores acuoso y vítreo, por lo tanto, su uso sistémico y tópico en las endoftalmitis causadas por Acremonium, Aspergillus o Fusarium podría, tal vez, ser de utilidad, lo mismo pudiera pensarse del uso del sulfametoxazol en las meningitis causadas por algunos de los hongos sensibles a él.

Lazo (por publicar) encontró que el sulfametoxol y la asociación sulfametoxazol cloranfenicol potencian marcadamente la acción anti C. albicans del fluconazol.

\section{REFERENCIAS}

ANTUNEZ, I.; JIMENEZ, C. HURTADO; J. GALEANO, J; BLANCO (1986). Asociaciones antifúngico-antibacteriano en el tratamiento de las micosis. III Reunión Conjunta de Micología, Badajoz. 163 pp.

COPELAND,B.E.; R.ROSVOLL.(1969). Sulfonamides and Aspergillosis. JAMA. 210 (13): 2398.

EILAM Y.: I. POLACHEK: G. BEN-GIGI; D. CHERNICHOVSKY. (1986). Activity of Phenotiazines against Medically Important Yeasts. Antimicrob. Agents Chemother. 31 (5): 834-836

GOODMAN GILMAN A.; L.S. GOODMAN; A. GILMAN. (1985). Goodman and Gilman's The Pharmacological Basis of Therapeutics. 7 ed. N. York. 1839 pp
KAMAYA,T. (1973). In vitro susceptibility of Candida albi cans and Candida stellatoidea to Sulphamethoxazole. Mycopath et Mycol. Applic. 51: 193-200.

LAZO. W.: L. FERRADA.(1986). Fármacos, antisépt cos y preservativos antimicóticos. Bol.Micol. 2 (4): 183-204.

NEGRONI,R. (1982). Caracteres farmacológicos de las drogas antitifúngicas empleadas en medicina. Primera parte. Bol.Micol. 1 (2): 60-67.

REYNOLDS, J.E.F. (1982). Martindale. The Extra Farmaco poeia. 28 ed. London. 2025 pp.

SAAG. M.S.; W.E. DISMUKES. (1988). Azole antifungal agents: Emphasis on new Triazoles. Antimicrob. Agents Chemother. 32 (1): $1-8$

\section{AGRADECIMIENTOS}

El autor desea expresar su gratitud a los Dres. Guido Pincheira y Luis Corcuera por haberle pemitido usar equipos e instrumental de sus laboratorios; al Dr. Eduardo Piontelli por haber facilitado las cepas de los hongos que su usaron en este estudio; a los Dres. Luis Ferrada a Igor Lemus por su ayuda decisiva para conseguir los fámacos que se emplearon y a los laboratorios Chile, Bayer, Beta, Ciba, Norgine, Parke Davis, Pasteur, Recalcine, Roche, Silesia, Squibb, Upjohn por haber proporcionado los fármacos que su utilizaron en este estudio. 


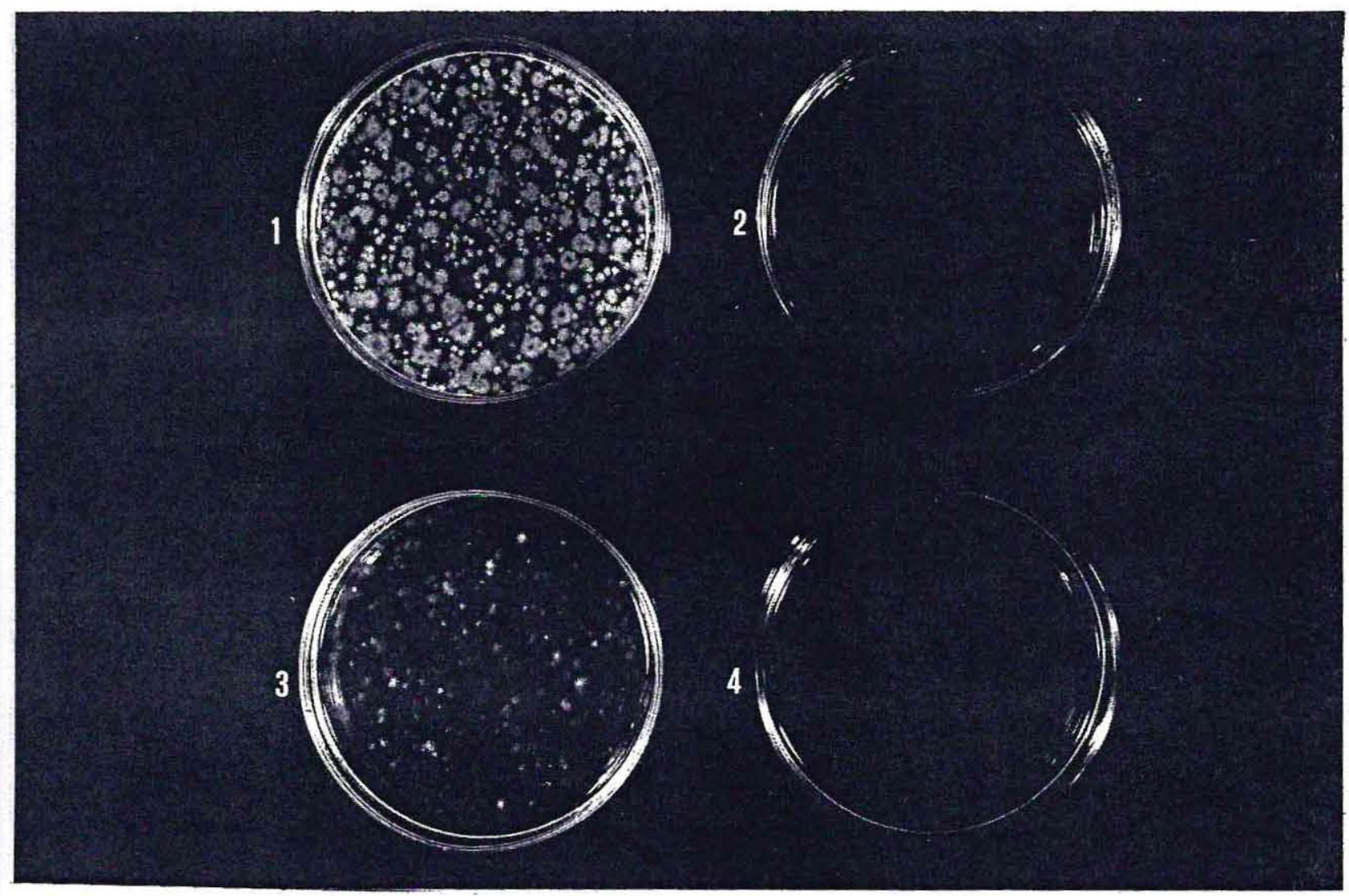

Foto 1. № 1. A. fumigatus, $48 \mathrm{hrs}$. de desarrollo a $35^{\circ} \mathrm{C}$. № 2. A. fumigatus, $48 \mathrm{hrs}$. de desarrollo a $35^{\circ} \mathrm{C}$ en medio con sulfametoxazol $100 \mathrm{ug} / \mathrm{ml}+$ fenilbutazona $20 \mathrm{ug} / \mathrm{ml}+$ etosuccimida $100 \mathrm{ug} / \mathrm{ml}$. No 3. F. solani $48 \mathrm{hrs}$. de desarrollo a $35^{\circ} \mathrm{C}$ № 4 . F. solani $48 \mathrm{hrs}$, de desarrollo a $35^{\circ} \mathrm{C}$ en medio con sulfametoxazol 100 $\mathrm{ug} / \mathrm{ml}+$ fenilbutazona $30 \mathrm{ug} / \mathrm{ml}+$ etosuccimida $100 \mathrm{ug} / \mathrm{ml}$. (fotos Lilio Yáñez). 